\title{
Estudio socioeconómico de las condiciones de vida de la población extranjera en España: el caso particular de Almería
}

\author{
Domingo Bonillo, Luis J. Belmonte e Isabel M. Román \\ Universidad de Almería (España)
}

\begin{abstract}
El objetivo de este trabajo consiste en presentar los importantes cambios demográficos que ha sufrido España, como consecuencia de la importante afluencia de extranjeros. En este contexto, la provincia de Almería se presenta como una de las más activas en el crecimiento del número de extranjeros, por lo que se considera conveniente la realización de un estudio socioeconómico sobre las condiciones de vida de este colectivo. Para ello, se ha diseñado un cuestionario de treinta preguntas, dividas en cuatro subepígrafes, con objeto de obtener información primaria sobre el contingente de extranjeros afincado en Almería. Así, tras definir el perfil del encuestado, las nacionalidades objeto de estudio y la distribución geográfica por municipios, se ha conformado una muestra representativa de 220 encuestas que ha permitido conocer mejor la realidad de este contingente. Finalmente, se constata como los resultados de nuestro estudio están en consonancia con las encuestas nacionales que se han llevado a cabo, aunque se presentan ciertas diferencias, especialmente en lo relativo al volumen de ingresos medios, características de su alojamiento y sector productivo en el que se emplean.
\end{abstract}

Palabras clave: Población extranjera, Almería, crecimiento poblacional, condiciones de vida.

A socioeconomic survey of the living conditions of the foreign population in Spain: A case study of the province of Almería. The aim of this paper is to provide an account of the major demographic changes that Spain has undergone as a result of the considerable increase of foreign population over the last few years. Since the province of Almería qualifies as one where the presence of foreign population is stronger, it then seems adequate to conduct a socioeconomic study of the living conditions of this group in this city. To this purpose, we designed a questionnaire consisting of thirty questions divided into four subsections, in order to elicit primary, first-hand information on a number of foreigners based in Almería. Thus, drawing on the profile of each respondent, their nationalities as well as their geographical distribution by municipalities, we have gathered a representative sample of 220 surveys which has provided us with realistic insights into this quota. An important conclusion emerging from our study is that, although the results reported here are consistent with other national surveys that have been previously carried out, a number of significant differences have been pinpointed, especially regarding the volume of income as well as the housing characteristics of the productive sector in which the foreigners in question are employed.

Key words: Foreign population, Almería, population growth, living conditions.

Correspondencia: Domingo Bonillo. Departamento de Economía Aplicada. Universidad de Almería. Ctra. La Cañada de San Urbano s/n, C.P. 04120. Almería (España). E-mail: dbonillo@ual.es 
En los últimos años, el rol migratorio de España ha sufrido un cambio radical, ya que ha pasado a convertirse en uno de los principales países receptores de inmigrantes, a diferencia de lo que ocurría en los cincuenta y sesenta. En este sentido, son muchas y complejas las causas que explican la situación actual, en la que España se ha consolidado como un receptor neto de extranjeros, con las consecuencias que ha tenido para nuestra sociedad (Dolado y Vázquez, 2008; Pajares, 2009 y De la Rica, 2010). En la última década el ritmo de crecimiento de extranjeros ha sido vertiginoso, ya que, si en 1998 este contingente representaba el 1.60\% de la población total de España (637.085), en 2010 dicho porcentaje asciende hasta el $12.22 \%$ del total de la población (5.75 millones de extranjeros). Véase la figura 1.

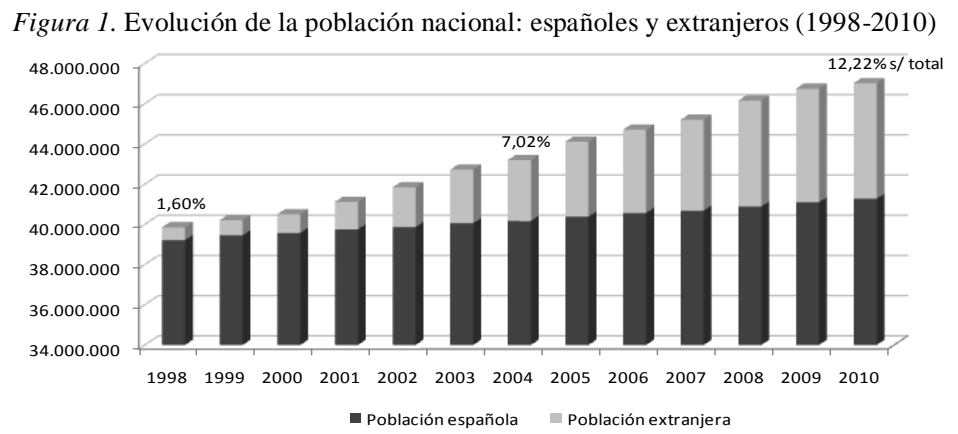

Fuente: INE y elaboración propia.

Dadas las perspectivas de envejecimiento de la población española (Belmonte et al., 2009), la ONU en el año 2000 aconsejó a España que admitiera anualmente a 240.000 extranjeros hasta el 2050, aunque dichas recomendaciones han sido ampliamente superadas en la última década. Con este incremento exógeno de la población española, se pensaba que se podría asegurar el pago de las pensiones, así como el mantenimiento del estado del bienestar. Sin embargo, la efectividad de ese razonamiento se ha truncado con la crisis económica, pues el número de desempleados se ha duplicado en poco más de dos años, incrementándose el número de subsidios y reduciéndose las afiliaciones.

De manera más concreta, se observa que la provincia de Almería presenta una tendencia de crecimiento de la población extranjera muy acentuada desde mediados de los noventa pasando de 13.260 en 1998 a 151.159 en 2010, es decir, este colectivo ha pasado de representar el $2.6 \%$ al $21.7 \%$ de la población almeriense. En este período la población extranjera de la provincia ha crecido un $1.040 \%$, mientras que la andaluza lo ha hecho en un $606 \%$ y la española en un $802 \%$.

Por otro lado, durante ese periodo, la tasa de variación anual acumulativa de la población extranjera residente en Almería se sitúa en el $22.5 \%$ frente al $17.7 \%$ y $20.1 \%$ 
de Andalucía y España, respectivamente. Esta significación es la más alta de todo el país por detrás sólo, de Alicante y Baleares, pues presenta un porcentaje del 21.9\%. A su vez, este porcentaje de significación supone casi el doble de la ponderación que tiene el colectivo de extranjeros en media nacional (12.22\%). Véase la figura 2.

Figura 2. Provincias con mayor porcentaje de extranjeros sobre la población total (2010)

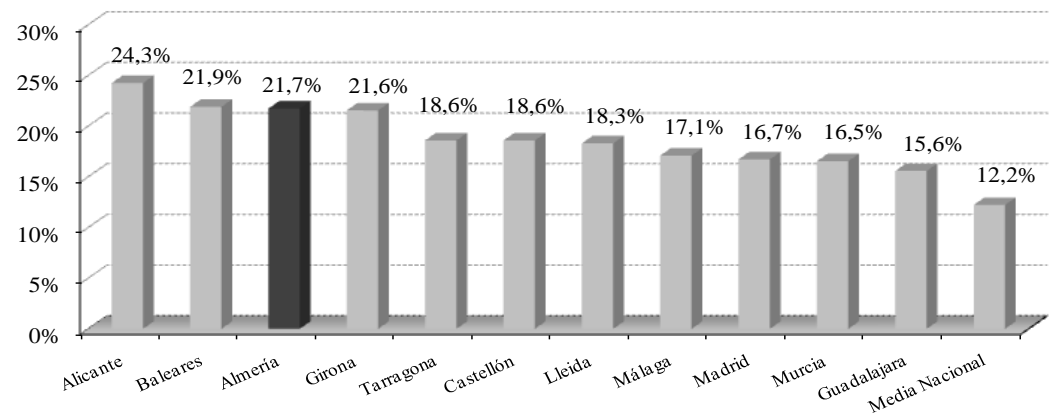

Fuente: INE y elaboración propia.

Estudio sociológico de las condiciones de vida de la población inmigrante en Almería: objetivos

Con la finalidad principal de obtener una visión sobre el nivel de bienestar que presenta el colectivo de extranjeros que residen en Almería, y conocer su proceso de inserción social y laboral, se ha diseñado un cuestionario con la intención de recabar información sobre las condiciones de vida y de disponibilidad de renta del colectivo de inmigrantes asentados en la provincia de Almería.

Los objetivos específicos que se persiguen con la realización del cuestionario individual consisten en:

- Averiguar la trayectoria migratoria que ha afectado a la vida del inmigrante, ahora residente en alguno de los municipios de la provincia de Almería.

- Presentar las condiciones de habitabilidad de las viviendas que este colectivo tiene a su disposición, prestando especial atención a la dotación de suministros y servicios básicos para una vida digna (agua corriente, electricidad y alcantarillado).

- Conocer el nivel de ingresos medios del inmigrante encuestado, así como el nivel de cobertura de los gastos periódicos de su hogar. Asimismo, cuando el encuestado se preste a ello, se recabará información relacionada con la situación administrativa en que se encuentra (en alta laboral, desempleado o trabajando en economía sumergida).

- Recabar información sobre la estructura de gasto de los hogares de extranjeros. En este sentido, se desea conocer la solvencia o dificultad con que este 
colectivo llega a fin de mes, adquiere su habitual cesta de la compra, la disponibilidad o no de tiempo y recursos para invertir en actividades lúdicas.

- Averiguar el grado de satisfacción personal de los inmigrantes residentes en la provincia de Almería en lo que respecta a la atención que reciben de las distintas Administraciones Públicas, de las asociaciones a las que puedan pertenecer o del resto del vecindario en el que radica su domicilio familiar.

\section{Perfil del inmigrante encuestado, tipo de muestreo y dimensión de la muestra}

El cuestionario que se ha diseñado está dirigido a una muestra representativa de inmigrantes que residen en alguno de los municipios que se han seleccionado en la provincia de Almería. En este sentido, se han seleccionado aquellos extranjeros que se encuentran empadronados en cualquiera de los ayuntamientos de la provincia de Almería, con especial atención a los que residan en los municipios de Almería, El Ejido, Roquetas de Mar, Vícar y Níjar debido a la concentración de extranjeros y diversidad de nacionalidades que éstos presentan.

Por otra parte, al igual que ocurre en la Encuesta Nacional de Inmigrantes (2007), se han considerado personas encuestables a todos aquellos inmigrantes mayores de 18 años que tienen un país de origen distinto al nuestro, pero tienen establecida su residencia habitual en España.

El tamaño de la muestra está íntimamente relacionado con la importancia que tiene cada nacionalidad en los municipios más importantes de la provincia de Almería. Así pues, según los datos suministrados por el INE, según el padrón municipal a enero de 2008, el total de extranjeros residentes en la provincia de Almería ascendía a 131.330. Asimismo, para determinar el número de encuestas a realizar en cada municipio, se ha tenido en cuenta el peso que representan los extranjeros en cada uno de ellos.

A la hora de determinar el ámbito geográfico en el que se va a pasar el cuestionario, así como la nacionalidad de los encuestados, se ha considerado conveniente restringir la muestra, tanto en número de municipios, como en número de nacionalidades. En la práctica, estas restricciones han supuesto:

1. Limitar a cinco el número de municipios en los que realizar el cuestionario, ya que agrupan el $63.2 \%$ del total de extranjeros de la provincia.

2. Limitar el número de nacionalidades objeto de análisis. Para ello se han agrupado las nacionalidades más importantes en siete conglomerados o clusters entre los que se distribuye el total de encuestas realizadas.

El muestreo se ha realizado de manera aleatoria, por conglomerados poblacionales, en cada uno de los municipios designados en la etapa de definición del trabajo de campo (Almería capital, El Ejido, Roquetas de Mar, Níjar y Vícar). La encuesta presencial directa se realizó entre los días 1 de enero y 28 de febrero de 2010, a extranjeros mayores de edad, residentes en Almería. 
Figura 3. Distribución de la población extranjera de la provincia de Almería por municipios
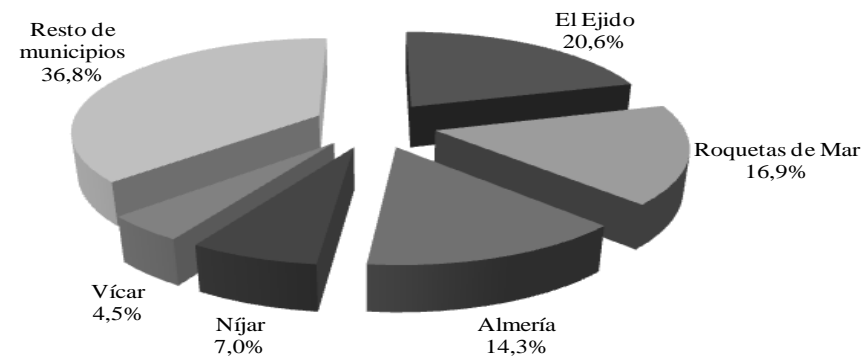

Fuente: INE y elaboración propia.

Dado el tamaño de la población objeto de estudio, se han realizado 220 encuestas, distribuidas entre los cinco municipios considerados. Para ello, se ha tenido en cuenta que el margen de error para el total de la muestra es del $+/-3.2 \%$, con un nivel de confianza del 95\%. Analíticamente, el tamaño muestral se ha obtenido aplicando la siguiente fórmula estadística para la proporción de poblaciones finitas:

$$
n=\frac{N}{1+\left(\frac{d}{z_{\frac{\alpha}{2}}}\right)^{2} \cdot \frac{(N-1)}{p \cdot q}}
$$

Donde, para la población de referencia, se considera que:

- " $n$ ” es el tamaño muestral mínimo, es decir, 220 encuestas.

- "N" es el tamaño de la población de referencia. En nuestro caso: 70.477 extranjeros.

- " "d" es el error muestral estándar. En nuestro caso: $6.41 \%$ (+/- 3.2\%).

- “Z $\alpha / 2$ ” es la variable tipificada, al nivel de significación $\alpha$, es decir, 1.96 para un nivel confianza del $95 \%$.

- “ $\overline{\mathrm{p}}$ " es la probabilidad promedio del suceso considerado, es decir, encuestar a un inmigrante en cualquiera de los cinco municipios considerados.

- “ $\overline{\mathrm{q}}$ " es la probabilidad promedio del suceso contrario al considerado, es decir, encuestar a un inmigrante fuera de cualquiera de los cinco municipios considerados.

- Para la desagregación muestral en función de las nacionalidades consideradas (subíndice i-ésimo) se ha utilizado la expresión análoga:

$$
\mathrm{n}_{\mathrm{i}}=\frac{\mathrm{N}_{\mathrm{i}}}{1+\left(\frac{\mathrm{d}}{\mathrm{z}_{\alpha / 2}}\right)^{2} \cdot \frac{\left(\mathrm{N}_{\mathrm{i}}-1\right)}{\mathrm{p}_{\mathrm{i}} \cdot \mathrm{q}_{\mathrm{i}}}}
$$


Así pues, la distribución de los encuestados en las zonas de la provincia de Almería refleja que el $34 \%$ de los cuestionarios se han pasado en el municipio de El Ejido, el 26\% en Roquetas de Mar, el 23\% en la zona de La Cañada y El Alquián, que se encuentran dentro del municipio capitalino, el $10 \%$ en Níjar y el restante $7 \%$ en el municipio de Vícar.

\section{RESULTADOS}

A finales de febrero de 2010 se concluyó la fase de tabulación de las 220 encuestas individuales con los siguientes resultados generales:

1. En función de la nacionalidad, el cuestionario individual se ha pasado a un total de 19 nacionalidades, organizadas en 7 grupos. El $42 \%$ de las mismas se ha realizado a extranjeros de origen magrebí, el $4 \%$ a ciudadanos de origen subsahariano, el $7 \%$ a personas residentes en alguno de los países que componían la Unión Europea de los 15 , el $28 \%$ a rumanos, el $5 \%$ a extranjeros con origen en alguno de los países de la extinta URSS (rusos y ucranianos), el 13\% a latinoamericanos residentes en Almería y el $1 \%$ a ciudadanos de origen chino.

2. Estos porcentajes se ajustan a la planificación inicial de la encuesta, salvo en el caso de extranjeros de origen magrebí, a los que se les ha realizado 92 encuestas en total, es decir, 3 menos de las que se habían planteado, debido a la dificultad de encontrar inmigrantes de esta nacionalidad en el municipio de Almería capital, dispuestos a ser entrevistados. Asimismo, se optó por incrementar el número de encuestas realizadas al colectivo de extranjeros de origen subsahariano, desde las siete encuestas inicialmente planificadas, hasta los nueve cuestionarios finalmente realizados.

En cuanto a la explotación estadística de los datos recabados mediante la encuesta individual, se ha decidido presentar sus resultados agrupados en función de la estructura propia del cuestionario. Así pues, a modo de esquema, las principales conclusiones son las siguientes:

1. Datos sociodemográficos recabados del encuestado. En este apartado del cuestionario se ha obtenido información relativa a la edad, sexo, nacionalidad, situación familiar, años que lleva residiendo en España y nivel de estudios.

En concreto, el perfil general del encuestado nos sugiere que se trata de un inmigrante, mayoritariamente varón, $57 \%$ de los encuestados, frente a un $43 \%$ de mujeres; con una mayoría, un $43 \%$, de entre 26 y 35 años. Se trata de personas que llevan residiendo en Almería entre 6 y 10 años (un 45\%), cuyo estado civil es el de casado, según lo afirman el 55\% de los encuestados, y además viven con su familia en España (un 81\%). Asimismo, en función del nivel de estudios, un $49 \%$ posee estudios secundarios, seguido por un $30 \%$ con estudios primarios. 
Por otra parte, la mayoría de los encuestados han tenido como primer destino migratorio la provincia de Almería, un 69\% de los mismos, pues había ciertas expectativas de lograr su tan ansiada estabilidad laboral (el 53\% de los encuestados se encuentra actualmente trabajando, frente al $36 \%$ que está en paro, especialmente como consecuencia de la crisis). La mayoría vive en casa alquilada o propia, un $88 \%$ y su ocupación principal se distribuye entre la agricultura (37\%) y la hostelería (30\%), donde prestan sus servicios sin ningún tipo de cobertura contractual (el 52\% no tiene contrato laboral) y cuando lo tienen se trata de un contrato por obra y servicio (30\%).

Asimismo, se trata de un colectivo bastante arraigado en la provincia de Almería, como lo demuestra que el $53 \%$ de los mismos ya posean la residencia permanente en España y hayan decidido permanecer en Almería. A pesar de su arraigo en la provincia, resulta escaso su nivel de asociacionismo, pues el $82 \%$ de los encuestados declara que no pertenece a ningún tipo de asociación.

A escala municipal, el perfil general de los extranjeros encuestados presenta ciertas particularidades. Por ejemplo, con relación al recorrido migratorio, en el caso de los residentes de El Ejido, un $84 \%$ pensó en Almería como su primer destino migratorio, frente a los extranjeros afincados en el municipio de Níjar, donde un $32 \%$ de los mismos declara haber vivido anteriormente en otro lugar de España.

En función de la nacionalidad, se destaca que un $77 \%$ de los marroquíes eligieron prioritariamente a Almería como primer destino de su proyecto migratorio, frente a los extranjeros con origen en el África Subsahariana, que han residido en otros lugares antes de asentarse en Almería (un 57\%).

En lo concerniente a las principales conclusiones de los distintos epígrafes del cuestionario, correspondientes al apartado de datos sociodemográficos, también se observan ciertas diferencias en función de las nacionalidades y del municipio de residencia. Así pues, en función del lugar donde residen los extranjeros, se observa cierta homogeneidad. En concreto, un 19\% de los encuestados en el municipio de El Ejido y un $20 \%$ de los residentes en Níjar declaran vivir en su lugar de trabajo. Por nacionalidades, los subsaharianos y los marroquíes presentan los porcentajes más altos de extranjeros que viven en sus centros de trabajo (un $14 \%$ y un $18 \%$, respectivamente).

Con relación a su situación laboral en España, el porcentaje más alto de parados se registra en Vícar, con el 53\% de los encuestados. En el otro extremo se sitúan los residentes en el municipio de Roquetas de Mar donde se registra el mayor porcentaje de ocupación, con el $62 \%$ de los encuestados. Sin embargo, la media provincial indica que la mayoría de los extranjeros encuestados (53\%) se encuentra actualmente trabajando, frente al $36 \%$ de los que declaran que se encuentran desempleados.

Por nacionalidades, el paro afecta mayoritariamente a los extranjeros de origen subsahariano, con un $57 \%$ de los encuestados, seguidos de los marroquíes, con el 
$49 \%$ de los mismos. Por otro lado, con pleno empleo se declara el colectivo de extranjeros procedente de Rusia, Ucrania y China.

Estos resultados se encuentran en consonancia con los publicados en la Encuesta Nacional de Inmigración-ENI (INE, 2009c), teniendo en cuenta el grupo de encuestas que se realizaron para la provincia de Almería. En este sentido, según esta encuesta nacional, el 57.6\% de los encuestados en 2007 se declararon trabajando, independientemente de su nacionalidad. Es posible que la diferencia entre los ocupados que refleja la ENI (57.6\%) y los que resultan de nuestro cuestionario individual se deba al momento en que se realizaron ambas encuestas. En el caso de la ENI las encuestas se llevaron a cabo a lo largo del año 2007, mientras que en nuestro caso, el horizonte temporal en el que se han llevado a cabo las encuestas ha discurrido desde el 1 de enero y el 28 de febrero de 2010, es decir, en plena crisis económica.

Figura 4. Situación laboral de los encuestados en la provincia de Almería

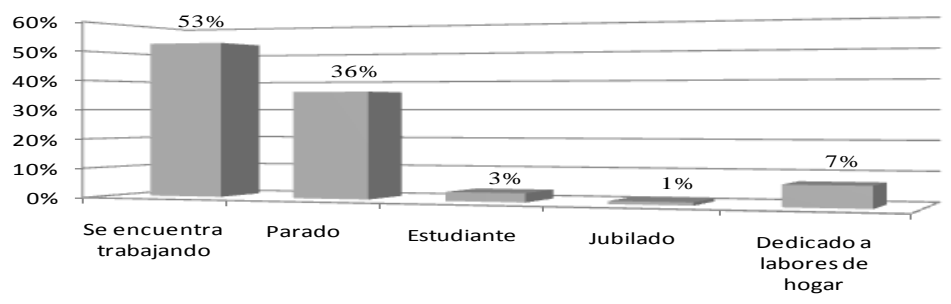

Fuente: elaboración propia a partir de encuesta directa

Figura 5. Sector productivo en el que prestan sus servicios los inmigrantes ocupados

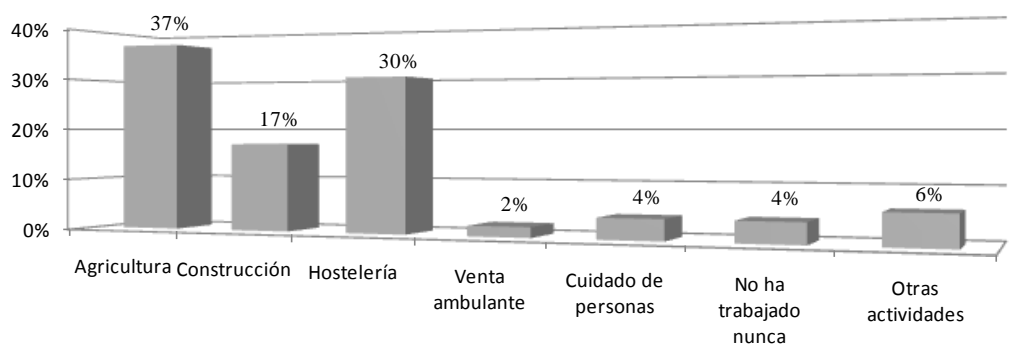

Fuente: elaboración propia a partir de encuesta directa

Asimismo, se detectan ciertas diferencias en función del municipio analizado. Así pues, en el caso de los extranjeros empadronados en los municipios de El Ejido y Níjar, una gran parte de ellos trabajan en la agricultura, un $66 \%$ y un $56 \%$, respectivamente. Sin embargo, los ocupados en la hostelería son mayoritarios en el caso del municipio de Almería capital (un 54\%) y muy representativos en el caso de Vícar, con un $46 \%$. 
En función de la nacionalidad, el colectivo de extranjeros del África Subsahariana se emplea mayoritariamente en la agricultura, con un $72 \%$ de los encuestados, seguidos de los inmigrantes de origen magrebí, con un 64\%. El resto de nacionalidades también se emplean con carácter preferente en la agricultura, a excepción de los ciudadanos de origen chino, que declaran dedicarse en su totalidad a la hostelería. En este sector trabajan mayoritariamente los ciudadanos de la Unión Europea (63\%), así como rusos y ucranianos (54\%).

Por el tipo de contrato, resulta destacable el elevado número de extranjeros encuestados que no tiene contrato laboral: un 52\% de media provincial, en consonancia con el $53.9 \%$ de encuestados por la ENI que no ha declarado ninguna modalidad contractual. Esta situación resulta especialmente importante en los municipios de Níjar (72\%) y Almería capital (58\%). Asimismo, resulta destacable el alto grado de precariedad del contingente de extranjeros, en el que sólo un 5\% de los mismos posee un contrato indefinido (16.1\% según la ENI de 2007). Por nacionalidades, los rumanos y búlgaros destacan por su alta vinculación contractual, con un $61 \%$ de los encuestados, seguidos por los subsaharianos y marroquíes, con un $57 \%$ de vinculación. Por otro lado, resulta diferenciador el hecho de que el $14 \%$ de los encuestados en el municipio de Roquetas de Mar sean autónomos.

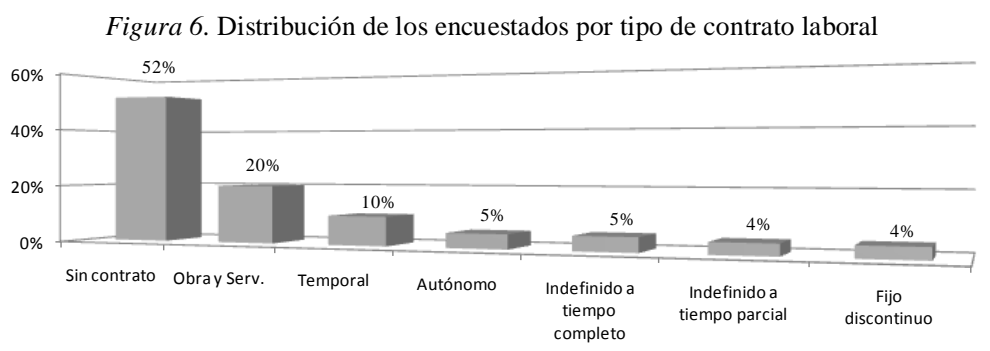

Fuente: elaboración propia a partir de encuesta directa

Por lo que respecta a la situación administrativa del colectivo de extranjeros residentes en la provincia de Almería, se constatan ciertas diferencias en función del ayuntamiento de empadronamiento. Destaca el caso de Vícar, El Ejido y el municipio capitalino con un alto porcentaje de extranjeros con residencia permanente, con un $60 \%$, $57 \%$ y un $54 \%$, respectivamente. La media provincial muestra que el $53 \%$ de los encuestados posee la residencia permanente en España. En este sentido, la Encuesta Nacional de Inmigración de 2007 (INE, 2009c) informa que el 19.4\% de los extranjeros encuestados en Almería poseen autorización de residencia permanente y que el $28.1 \%$ ostenta la autorización de residencia temporal, que junto a los extranjeros que poseen la 
tarjeta de residencia comunitaria (3.7\%) supone que un $51.2 \%$ de extranjeros, según la ENI, tiene autorización de residencia legal en Almería.

2. Nociones objetivas sobre la calidad de vida de los encuestados. En esta sección se ha recabado información sobre la higiene, dimensión y habitabilidad de las viviendas donde residen los inmigrantes asentados en los municipios más importantes de la provincia de Almería. Asimismo, esta sección del cuestionario incluye preguntas tendentes a recopilar información sobre la titularidad de la vivienda, disponibilidad de electrodomésticos que faciliten una vida confortable al hogar del inmigrante, así como si se dispone de una adecuada red de suministro de agua potable, alcantarillado público, etc. En la práctica, las declaraciones de los encuestados han sido validadas por la observación directa del encuestador, siempre que ha sido posible.

En primer lugar sobre el nivel de higiene de las viviendas, la calificación en general es muy buena y buena, con un $83 \%$ de las respuestas. A este alto porcentaje contribuye la buena percepción de las dimensiones de de la vivienda (72\% de los encuestados), la escasez de goteras $(77 \%)$, la proximidad al centro urbano $(81 \%$ la declaran buena y muy buena) y la buena comunicación viaria (78\%).

Por municipios, todos en general tienen una opinión de buena y muy buena sobre el grado de higiene de su vivienda habitual, aunque resulta destacable que en el municipio de El Ejido un 30\% de los encuestados declaran que dicha situación es regular o mala. A cierta distancia, con el $16 \%$ de los extranjeros encuestados, se encuentra el municipio de Níjar.

Sin embargo, en función de la nacionalidad de los encuestados, las diferencias se hacen más patentes. En concreto, el $50 \%$ de los ciudadanos de nacionalidad china manifiestan una situación regular en cuanto a la higiene de su vivienda, seguidos de los encuestados del África Subsahariana, con el 29\% de los encuestados, y de $23 \%$ de los marroquíes. En cuanto a la dimensión de la vivienda, los encuestados de África Subsahariana, con un $43 \%$ de las respuestas, la califican de regular, seguidos por los marroquíes, con el 29\%, también de regular. Junto con los problemas de goteras, proximidad al centro urbano y comunicación viaria, estos dos colectivos son los que presentan un perfil con preponderancia a la regularidad.

Por lo que respecta a la posesión de electrodomésticos y otros bienes de consumo duradero ( $\mathrm{TV}$, radio, teléfono y coche), predomina la disponibilidad de los mismos, en un porcentaje superior al $90 \%$ de los encuestados. En concreto, en cuanto a la disponibilidad de electrodomésticos, el 93\% de los encuestados en la provincia de Almería manifiesta poseerlos (el $98 \%$ tiene TV o radio y el $97 \%$ tiene teléfono). Sin embargo, las respuestas afirmativas decaen cuando se pregunta por la disponibilidad de vehículo (coche o moto), en el que sólo el 59\% manifiesta poseerlo. Por lo que respecta a los traslados en bicicleta, un 33\% de los extranjeros afincados en la provincia de Almería 
utiliza este medio de transporte, a pesar de que algunos de ellos disponen de automóvil o moto.

En relación a la titularidad de la vivienda principal, sólo la reconoce un 34\% de los inmigrantes encuestados. Este porcentaje asciende al $44.3 \%$ en el caso de los extranjeros encuestados por el INE a la hora de realizar la Encuesta Nacional de Inmigración de 2007. Asimismo, los extranjeros que declaran residir en una vivienda cedida o alquilada, según la ENI, ascienden al 55.2\% de los encuestados. En nuestro caso, el estudio que hemos llevado a cabo a través de cuestionario indica que el $64 \%$ de los mismos reside en una vivienda cedida o alquilada. Dentro de este el $41 \%$ ha declarado que el alquiler lo han generado directamente los extranjeros interesados.

Por ayuntamiento, el porcentaje de posesión oscila entre el $12 \%$ de Níjar y el $47 \%$ de Vícar. Por nacionalidad, se puede mencionar que los inmigrantes de la Unión Europea de los 15 manifiestan poseer vivienda de forma mayoritaria (un 56\%), seguidos de los chinos (50\%). Por el contrario, el $86 \%$ de los encuestados con origen en África Subsahariana manifiestan que no son propietarios de una vivienda en España.

En referencia al grado de satisfacción con los servicios recibidos en ambulatorios y hospitales, la mayoría de los encuestados (56\%) declara que es buena y muy buena. Con una calificación de regular se encuentra un $25 \%$ de los mismos, mientras que el $9 \%$ la califica de mala y muy mala.

En función del municipio o ayuntamiento de empadronamiento, el dato más relevante lo constituyen los inmigrantes empadronados en Almería capital, donde el 92\% aprecia muy positivamente su satisfacción con los servicios sanitarios recibidos. A continuación se sitúan los empadronados en Níjar, con el $80 \%$ de los encuestados que califican tales servicios como buenos y muy buenos, así como Roquetas de Mar (con un $67 \%$ de las observaciones). Por el contrario, las peores calificaciones de la atención sanitaria prestada a los inmigrantes se encuentra en El Ejido, donde el $16 \%$ de los extranjeros declara que el servicio recibido es malo o muy malo.

Por nacionalidades, destaca el $78 \%$ de iberoamericanos, seguidos del $72 \%$ de subsaharianos, con una opinión positiva de la atención sanitaria recibida. Mientras que con una opinión mala y muy mala se sitúa el $20 \%$ de los inmigrantes rusos y ucranianos.

Por otra parte, en cuanto a la calificación del grado de satisfacción con los servicios recibidos por el Ayuntamiento por parte de los encuestados, en general, hay una opinión positiva. En concreto, así lo piensa el $70 \%$ de los encuestados, que declaran que es buena y muy buena. Mientras que sólo el $6 \%$ cree que es mala y muy mala. En función del municipio de residencia, las mejores calificaciones las recibe el Ayuntamiento de Roquetas de Mar, donde el $81 \%$ de los encuestados está muy contento (opinión buena y muy buena) con los servicios prestados por el consistorio. Le sigue el Ayuntamiento de El Ejido (con el $79 \%$ de los encuestados) y el consistorio capitalino, con el $78 \%$ de los mismos. Sin embargo, las opiniones más negativas, en cuanto a la 
gestión municipal se centran en Níjar, en el que el 12\% de los encuestados manifiesta una opinión negativa.

Relacionado con el grado de satisfacción con otras actuaciones de la Administración, la calificación entre muy buena y buena asciende al 54\% y la negativa, entre mala y muy mala al 14\%. Asimismo, el $32 \%$ de los encuestados califica como regular el resto de actuaciones de la Administración. Por ayuntamientos, los mejores resultados los vuelve a obtener el municipio de Roquetas de Mar, con un $76 \%$ de los encuestados, seguidos de Vícar, con el $67 \%$ de los mismos.

3. Nociones subjetivas sobre la situación económica del hogar. En esta sección se ha recabado información relativa a la renta disponible de las familias de los encuestados, así como sobre la solvencia que esos hogares presentan a la hora de enfrentarse a sus gastos cotidianos. Se trata de uno de los apartados que más evidencias ofrece sobre las condiciones de vida de los hogares de los encuestados. En concreto, se pregunta por la facilidad o dificultad con que los inmigrantes pueden acceder a una cesta de la compra adecuada, disfrutar de vacaciones al menos una semana al año, así como la facilidad de llegar a fin de mes con el nivel de ingresos disponibles.

En concreto, a la pregunta sobre la posibilidad de permitirse una comida de carne, pollo o pescado al menos cada dos días, en general los inmigrantes encuestados han respondido en forma positiva en un $84 \%$ de los casos. Esta cifra aumenta en el caso de los residentes en el municipio capitalino, con el $96 \%$ de los datos observados. Sin embargo, en función de la nacionalidad las respuestas difieren sensiblemente. Así pues, el $100 \%$ de los inmigrantes del África Subsahariana declaran que no pueden permitirse dicho gasto, frente al $100 \%$ de residentes de origen chino, iberoamericano o de la UE [15], que afirman que sí pueden permitírselo.

En cuanto a la posibilidad de mantener una temperatura adecuada en el hogar, el $59 \%$ de los encuestados afirma que puede permitirse dicho gasto, frente al $41 \%$ que no pueden afrontar adecuadamente los meses fríos o calurosos. Por municipios, en Vícar existe el mayor porcentaje de encuestados que se lo pueden permitir, con un $80 \%$, seguidos por el $72 \%$ de los que viven en Roquetas de Mar. Por nacionalidad, responden positivamente el $100 \%$ de inmigrantes de origen chino y el $80 \%$ de rusos y ucranianos. Los que no se lo pueden permitir, están representados en primer lugar por los de África Subsahariana con el $86 \%$, seguidos por el $55 \%$ de inmigrantes marroquíes.

El último apartado de la vigésima pregunta del cuestionario hace referencia a la posibilidad que tienen los inmigrantes para permitirse, al menos, una semana de vacaciones al año. En este sentido, el $51 \%$ de los encuestados declaran que se la pueden permitir, frente al $49 \%$ que no puede. Por municipio de residencia, los que viven en Vícar, con el $67 \%$ de las observaciones, se lo permiten, seguidos por los empadronados en Roquetas de Mar con el 65\%. Por nacionalidad, los de China con el $100 \%$ se lo permiten, seguidos por el $95 \%$ de los inmigrantes de la UE [15]. De los que no se lo 
pueden permitir destaca el colectivo de inmigrantes de origen subsahariano, con el $86 \%$ de los encuestados, seguidos por el $75 \%$ de los inmigrantes de origen iberoamericano.

Por término medio, el $49 \%$ de los encuestados en la provincia de Almería declaran unos ingresos medios mensuales de entre $500 €$ y $1.000 €$. Para este mismo tramo retributivo, la Encuesta Nacional de Inmigración indica que el $25.3 \%$ de los encuestados en 2007 declararon encontrarse en ese tramo. Sin embargo, los porcentajes se invierten en el caso del tramo de $1.000 €$ a $2.000 €$, pues nuestra encuesta individual indica que sólo el 19\% de se encuentra en ese tramo. En cambio, para 2007, la ENI indicaba que el $22.1 \%$ de los encuestados en Almería cobraba más de $1.000 €$ y menos de 2.000€. Una vez más, la crisis económica es la que explicaría el descenso en este porcentaje.

La media provincial difiere significativamente con ciertos municipios. Así pues, los extranjeros que viven en Vícar declaran, con un $47 \%$ de los datos recabados, que perciben unos ingresos entre $1.000 €$ y $2.000 €$, frente al $32 \%$ de inmigrantes que residen en Níjar, que manifiestan unos ingresos inferiores a $200 €$ y donde además el $20 \%$ de los mismos percibe entre $200 €$ y $500 €$. Por nacionalidad, los subsaharianos presentan los ingresos más bajos, pues el $21 \%$ de los mismos declara ganar menos de $200 €$ y el $58 \%$ de esa nacionalidad indica que su nivel de rentas está en torno a los $200 €$ y $500 €$. Sin embargo, el nivel de ingresos mensuales más altos corresponde a los inmigrantes con origen en la UE [15], en los que el $44 \%$ de los mismos declara ganar entre $1.000 €$ y $2.000 €$ y el $24 \%$ de este clúster de nacionalidad gana más de $2.000 €$.

Relacionado con el poder adquisitivo que manifiestan los inmigrantes, se trata de recabar información sobre el nivel de dificultad para llegar a fin de mes todo el grupo familiar. En este sentido, el 34\% de los encuestados en la provincia de Almería manifiesta que llega a fin de mes con mucha dificultad, frente al $9 \%$ que declara que lo hace con facilidad y mucha facilidad.

Por municipios, los que viven en El Ejido, con el 69\% de los declarantes, se presentan como los que más dificultad tienen para llegar a fin de mes, frente a los que viven en Vícar, donde el $20 \%$ de los encuestados salvan con facilidad el mes. Les siguen los inmigrantes residentes en Roquetas de Mar, donde el 19\% también llega a fin de mes con facilidad.

Por nacionalidad, el $100 \%$ de los nacidos en el África Subsahariana llegan con mucha dificultad a fin de mes, entre otras razones porque son los que tienen el nivel de ingresos más bajos. Le siguen los marroquíes con el $48 \%$ de los encuestados. Por el contrario, el $43 \%$ de rusos y ucranianos, llegan con facilidad y mucha facilidad a fin de mes.

Por otro lado, en la pregunta múltiple vigésimo tercera se trataba de averiguar si los hogares de los inmigrantes encuestados logran mantenerse exclusivamente con los ingresos familiares o perciben alguna otra ayuda económica. En este sentido, la media 
provincial indica que el $77 \%$ de los encuestados logra mantenerse exclusivamente con sus ingresos, mientras que el resto recurre también a las ayudas económicas de la Junta de Andalucía (el 16\% de los encuestados), del Ayuntamiento en el que se encuentran empadronados (4\%) y de asociaciones sin ánimo de lucro (4\%).

Por lugar de residencia, los inmigrantes de Níjar son los que más ayuda externa reciben, con el $40 \%$ de los encuestados. Los que reciben ayuda de la Junta de Andalucía viven, en un 25\%, en Roquetas de Mar, mientras que en El Ejido, el 7\% de los inmigrantes reconoce ayuda económica de su ayuntamiento. Además en El Ejido las asociaciones sin ánimo de lucro prestan ayuda financiera al $8 \%$ de los encuestados.

Por otra parte, en ciertas actividades, especialmente las relacionadas con el sector agrícola, el empresario suele contribuir al sostenimiento familiar mediante ayudas en especie. Así lo reconocen el $17 \%$ de los extranjeros encuestados en la provincia de Almería, que llegan al $44 \%$ en el caso de los inmigrantes que residen en el municipio capitalino y un $41 \%$ en el caso de los iberoamericanos. Asimismo, el abastecimiento de alimentos en la granja o en la empresa en la que trabajan es habitual en un $14 \%$ de los casos, porcentaje que asciende al $24 \%$ en el caso de Níjar.

En cuanto al nivel de endeudamiento familiar, el $27 \%$ de los encuestados reconoce que tiene cuentas bancarias con saldos negativos, el $29 \%$ tiene algún pago pendiente de préstamos comerciales y compras a plazo, el $19 \%$ que mantiene deudas con los comercios en los que compra habitualmente, el 32\% tiene alguna deuda con familiares y vecinos y el $38 \%$ se ha retrasado en el pago de los recibos de agua, luz, teléfono.

Figura 7. Nivel de endeudamiento familiar. Media provincial

¿Posee cuentas bancarias con saldos negativos?

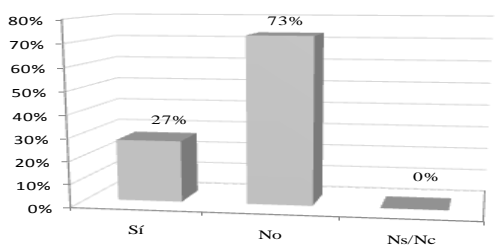

¿Mantiene deudas con los comercios habituales?

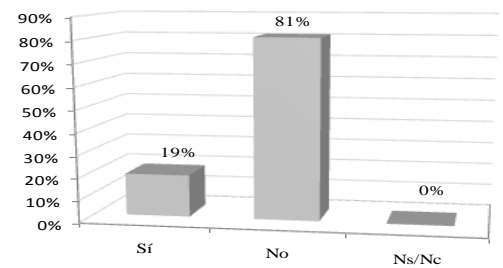

¿Tiene algún pago pendiente por préstamos?

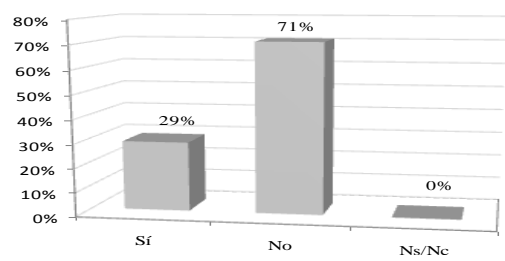

¿Mantiene deudas con familiares o vecinos?

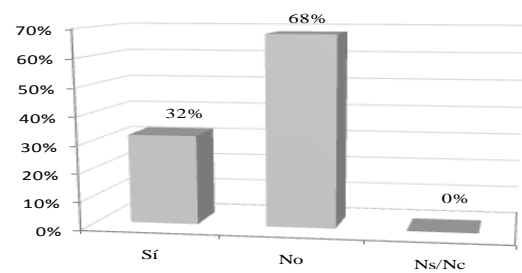

Fuente: elaboración propia a partir de encuesta directa 
En función del municipio de residencia, en Níjar se encuentra el porcentaje más alto de encuestados con cuentas bancarias con saldos negativos, el 44\%. Mientras que en El Ejido, el 47\% reconoce que tiene cuotas pendientes de préstamos comerciales y compras a plazo. Asimismo, en este municipio también se encuentra el porcentaje más alto de encuestados con deudas en los comercios habituales y con familiares y vecinos, con un $32 \%$ y un $49 \%$, respectivamente. También en el Ejido, el $51 \%$ de los extranjeros encuestados declara haberse retrasado en el pago de los recibos de agua, luz y teléfono.

Por nacionalidad, los inmigrantes de Rumania y Bulgaria, con el 34\% de los encuestados, reconoce que tiene cuentas bancarias son saldos negativos. Los extranjeros de origen latinoamericano, con el $56 \%$ de las observaciones, reconoce que tiene cuotas de préstamos comerciales y compras a plazo pendientes. Los nacidos en Marruecos, en un 23\%, afirman que mantiene deudas con los comercios en los que compran habitualmente. Finalmente, el $71 \%$ de los subsaharianos afirman que mantienen alguna deuda con sus familiares y vecinos y en ese mismo porcentaje también han tenido algún retraso en el pago de los recibos de agua, luz, teléfono.

La última pregunta de esta sección corresponde a la dificultad sobrevenida que ha supuesto la crisis económica. En concreto, se pregunta por la variación de ingresos que han podido experimentar los encuestados en los últimos doce meses. En este sentido, el $25 \%$ afirma no haber sufrido variación alguna en su nivel de ingresos, en el último año, por lo que el $75 \%$ sí lo ha notado. Asimismo, el $40 \%$ manifiesta que el descenso en sus ingresos se ha debido a la pérdida de su empleo, el $24 \%$ declara que ha sido como consecuencia de la reducción de la jornada laboral, el 14\% por otros motivos (menos clientes, menos turistas, etc.) y el $1 \%$ por la imposibilidad de trabajar como consecuencia de una enfermedad o incapacidad temporal.

Por ayuntamiento, destaca Roquetas de Mar que con el $61 \%$ de los encuestados no han notado un descenso de los ingresos frente al El Ejido que solo el $1 \%$ no lo ha notado. Por nacionalidad, el $44 \%$ de los nacidos en países de la Unión Europea de los 15 seguidos por el $37 \%$ de los nacidos en Rumania y Bulgaria. Los que lo han notado por la pérdida de empleo están en primer lugar con el $48 \%$ los nacidos en Iberoamérica, por la reducción de la jornada laboral con el 50\% los de China seguidos por el $40 \%$ de los nacidos en Rusia y Ucrania.

4. Entorno y perspectivas futuras de los encuestados. En esta sección se ha recabado información sobre las perspectivas futuras del colectivo de extranjeros residentes en la provincia de Almería. Así pues, las cuatro últimas preguntas del cuestionario se han encaminado a averiguar si Almería es el final de su proyecto migratorio, el grado de integración en la sociedad almeriense, las reivindicaciones más importantes a las distintas Administraciones Públicas y, finalmente, se pregunta por los planes de futuro del inmigrante encuestado. 
En la primera pregunta de esta sección, cuestión número veintisiete, se trata de conocer si Almería es un destino finalista para el proyecto migratorio del encuestado. En este sentido, el $69 \%$ reconoce que si lo es, aunque este porcentaje asciende al $92 \%$ cuando se trata de encuestados que residen en el municipio de Níjar y al $80 \%$ si se trata de El Ejido. En función de la nacionalidad, los marroquíes, con el $77 \%$ de los encuestados, y los rumanos y búlgaros, con el $72 \%$, son los más convencidos en que la provincia de Almería es el final de su trayecto migratorio.

En cuanto a los motivos que influyeron en la elección de la provincia de Almería como destino para vivir, el $42 \%$ reconoció que fue por reagrupamiento familiar, seguido del $26 \%$ que indicaron que fue como consecuencia de tener amigos y conocidos en Almería. Sin embargo, también existen otras razones de menor importancia, como era el hecho de que en Almería parecía haber trabajo (18\% de los encuestados), por las facilidades para regularizar su situación administrativa $(7 \%)$, por proximidad geográfica (5\%) y sólo el $2 \%$ vino a Almería por afinidad cultural y lingüística.

La importancia de estos motivos se presenta desigual según el municipio que se considere. Mientras que en El Ejido, Vícar y Almería capital el motivo principal fue el reagrupamiento familiar, en Roquetas de Mar y Níjar el motivo principal ha sido la existencia de amigos y/o conocidos.

En función de la nacionalidad, el $100 \%$ de los nacidos en China llegó a Almería con motivo de la reagrupación familiar, seguidos por el $54 \%$ de los nacidos en Marruecos. Por tener contactos y amigos a la hora de venir a Almería destaca el $43 \%$ de los nacidos en África Subsahariana, seguidos por el $40 \%$ de los rusos y ucranianos. Entre aquellos que vinieron a Almería porque creían que era una buena oportunidad destaca el contingente de rumanos y búlgaros, con el $22 \%$ de los encuestados, seguidos del $20 \%$ de los rusos y ucranianos.

Con relación al sentimiento de integración que sienten los inmigrantes afincados en alguno de los municipios de Almería que se han analizado, las respuestas mayoritarias se centran en torno a los que se consideran "bastante integrado" y "muy integrado", que suman el 55\%, seguidos por el $24 \%$ que declara sentirse "algo integrado", el 15\% "poco integrado" y el 6\% "nada integrado".

Por municipios, las respuestas se presentan bastante dispares. Mientras que en El Ejido sólo el 33\% se considera muy y bastante integrados, en el municipio de Almería capital el $84 \%$ de los encuestados sí se encuentran muy y bastante integrados. Almería y Vícar son los dos únicos municipios donde ningún inmigrante se ha manifestado como "nada integrado". Por su parte, en Roquetas de Mar y Níjar lo más destacado es el porcentaje de inmigrantes "nada integrados", con el 6\% y el 4\%, respectivamente.

Por nacionalidad, los subsaharianos los que se manifiestan menos integrados, con un $86 \%$ de los mismos que se declara "poco integrado", a diferencia del 100\% de chinos encuestados, que se consideran "bastante integrados". Por su parte, los 
ciudadanos de la UE [15] se dividen entre el 75\% que se consideran "muy integrados", "bastantes integrados" y "algo integrado", a partes iguales, seguidos por el $13 \%$ que se consideran "poco integrados" y el 12\% "nada integrados". Asimismo, los nacidos en Rusia y Ucrania, en un $50 \%$, se sienten mayoritariamente "muy integrados", al igual que los inmigrantes de origen latinoamericano, con el 56\% de los encuestados. A menor escala de integración se manifiesta el $40 \%$ de los rumanos y búlgaros (40\% bastante integrados). Sin embargo los extranjeros de origen marroquí se consideran "algo integrados" en un 34\% de los casos.

Los que sienten que no están "nada integrados" están compuestos por el $12 \%$ de extranjeros procedentes de la UE [15], el 10\% de marroquíes, el 5\% de rumanos y búlgaros y el $3 \%$ de iberoamericanos.

Con relación a las exigencias que los inmigrantes le realizarían a las distintas Administración Públicas, los encuestados ordenaron y seleccionaron sus tres primeras peticiones. En concreto, el $38 \%$ de los encuestados en la provincia de Almería considera que la primera exigencia es la obtención de un trabajo digno, seguido del $18 \%$ de extranjeros a quienes le interesaría un mejor acceso a la vivienda. Por último, la tercera reivindicación del colectivo de extranjeros fue la de solicitar una mayor facilidad para regularizar su situación administrativa, con el 13\% de los encuestados.

Por último, a propósito de los planes de futuro del inmigrante encuestado, el $48 \%$ de los inmigrantes afirman que han decidido quedarse en Almería, seguidos por el $26 \%$ que desean volver a su país de origen, el $20 \%$ que no tiene claro le deparará el futuro, el $5 \%$ que se marcharía a otro lugar de España y sólo el 1\% que tiene decido irse a otro país. Estos porcentajes difieren sensiblemente de los que se obtuvieron por la ENI en 2007, pues en aquellas fechas sólo el 3.7\% de los encuestados se planteaba volver a su país en los próximos cinco años, mientras que el $91.7 \%$ de los encuestados indicaba que deseaba permanecer en España.

Por municipios, los que viven en Vícar tienen decidido quedarse en Almería, en un $73 \%$ de los casos observados. En el caso de Níjar, el $40 \%$ tiene pensado volver a su país y en el caso de Roquetas de Mar, el $42 \%$ no tiene decidido que hará con su futuro.

Por nacionalidades, los subsaharianos, con un $58 \%$ de los encuestados, no tiene decidido que hará en el futuro. Asimismo, un 50\% de los extranjeros encuestados con origen en países de la UE [15] se quedarán en Almería, al igual que los rusos y ucranianos. Este porcentaje asciende al $63 \%$ en el caso de los ciudadanos de origen latinoamericano, así como los marroquíes, en un 56\% de los encuestados. Sin embargo, un $32 \%$ de rumanos y búlgaros declara que volverá a su país, junto con un $28 \%$ que aún no sabe qué hará en el futuro. 


\section{CONCLUSIONES}

Se ha comprobado cómo, en la última década, los extranjeros con permiso o tarjeta de residencia en nuestro país han experimentado un crecimiento de tal magnitud que ha sobrepasado las previsiones más optimistas, sin precedentes en los países de nuestro entorno, haciendo cambiar la pirámide poblacional española y suministrando al mercado de trabajo la mano de obra que precisaba en la época de fuerte expansión económica. Así, más del $62 \%$ del incremento experimentado por la población residente en España, entre el año 2000 y el 2010, se debió a la población extranjera.

Respecto a la incidencia de la población activa extranjera en Andalucía y particularmente en la provincia de Almería, hemos constatado que la población inmigrante ha sido una pieza básica para la recuperación económica de Andalucía y sobre todo de Almería, al aportar mano de obra que demandaba la agricultura intensiva desde hace décadas y los sectores de la construcción y de servicios durante el presente siglo.

Hemos constatado que la población inmigrante preocupa a la sociedad andaluza y en mayor medida a la almeriense, al haber experimentado un crecimiento superior a cualquier otra provincia en la última década y suponer una quinta parte de la población que reside en la provincia de Almería. Aunque ha supuesto un dinamismo para nuestra economía y un fortalecimiento para nuestra población, en la actual época de crisis, en la que hay excedente de mano de obra, representa un problema por la posible competencia con la población autóctona. Sin embargo, entendemos que al no estar estrictamente vinculada la inmigración a los ciclos económicos y dado que la mayor parte del colectivo inmigrante permanece en Almería durante las recesiones económicas, hay que propiciar un clima que favorezca su integración, ayudándoles a salir de la situación de marginación y pobreza en la que una parte importante se encuentra.

Del estudio socioeconómico, las conclusiones más relevantes que se desprenden del cuestionario individual son las siguientes:

- En cuanto a los datos sociodemográficos del inmigrante encuestado, constatamos que se trata de una población joven, con mayoría de varones, que ha llegado a Almería hace menos de diez años, confirmando lo expuesto con anterioridad sobre la evolución de dicho colectivo en la provincia de Almería y en concordancia con los resultados de la ENI sobre los flujos migratorios. Además, en su mayoría dicen estar casados y residiendo con sus familias. La mitad de los encuestados manifiestan poseer una educación secundaria. Se trata de un colectivo con tarjeta de residencia permanente, que está bastante arraigado en la provincia de Almería y está trabajando en la agricultura y hostelería principalmente, con una alta precariedad laboral. Estas características presentan diferencias notables en función de la nacionalidad de procedencia del inmigrante. 
- En cuanto a las nociones objetivas sobre la calidad de vida de los encuestados. En general, las viviendas de los inmigrantes presentan en unos porcentajes muy altos buenas condiciones higiénicas, con unas dimensiones aceptables, sin goteras y próximas al casco urbano. No obstante, en el municipio de El Ejido casi un tercio de los encuestados declaran que la situación de su vivienda es regular o mala; y atendiendo a la nacionalidad, los chinos, subsaharianos y marroquíes manifiestan una situación regular en la higiene de su vivienda. Más del $90 \%$ posee electrodomésticos y el 59\% dispone de vehículo para desplazarse, aunque hay notables diferencias en función de la nacionalidad. Algo más de la tercera parte de los encuestados dicen ser propietarios de la vivienda principal, residiendo en vivienda alquilada por ellos mismos el $41 \%$. Son mayoría $(56 \%)$ los que califican la asistencia sanitaria recibida como buena o muy buena, frente a los que la califican como mala o muy mala (9\%), con diferencias apreciables según nacionalidades y el lugar de residencia. También es bueno o muy bueno el grado de satisfacción que tienen los inmigrantes por los servicios recibidos de los ayuntamientos y de otras administraciones públicas (70\%).

- Por lo que respecta a las nociones subjetivas sobre la situación económica del hogar, aunque el $84 \%$ de los encuestados manifiestan poder permitirse una comida de carne, pollo o pescado al menos cada dos días, hay grandes diferencias entre nacionalidades; mientras la totalidad de los subsaharianos dicen no poder permitírselo. En cuanto a la posibilidad de mantener una temperatura adecuada en el hogar, el $59 \%$ de los encuestados dicen poder permitirse dicho gasto, aunque sólo declaran poder permitírselo el $14 \%$ de los subsaharianos. En lo referente a la posibilidad de disfrutar al menos de una semana de vacaciones al año, sólo el $51 \%$ de los encuestados manifiestan que se lo pueden permitir. Atendiendo al nivel de ingresos medios mensuales, los ingresos mensuales de la mitad de los encuestados se sitúan en el intervalo entre 500 y 1000 euros, con diferencias notables entre municipios. Dicen tener mucha dificultad para llegar a fin de mes con los ingresos familiares de que disponen el $34 \%$ de los entrevistados, frente al $9 \%$ que declara que lo hacen con facilidad. El $77 \%$ de los inmigrantes dicen que se mantienen sólo con los ingresos de su trabajo, mientras que el resto recurre a las ayudas económicas de las administraciones públicas y asociaciones, con diferencias apreciables en función de la nacionalidad y la actividad que desempeñan.

- Por último, en cuanto al entorno y perspectivas futuras de los encuestados, el $69 \%$ de los encuestados manifiesta que la provincia de Almería es el destino final de su proyecto migratorio. Los motivos por los que eligieron la provincia de Almería como destino para vivir, en orden de importancia, dicen ser: el reagrupamiento familiar, así como la existencia amigos y conocidos que residían en Almería. Declaran que se sienten bastante integrados y muy integrados en la sociedad almeriense el $55 \%$ de los inmigrantes, frente al $21 \%$ que dicen estarlo poco o nada, con diferencias apreciables entre municipios de residencia y nacionalidad. Demandan a las administraciones 
públicas, fundamentalmente, un trabajo digno el 38\%, mejor acceso a la vivienda el $18 \%$ o que se regularice su situación en España el $13 \%$.

Finalmente, se ha comprobado que nuestra encuesta guarda una alta sintonía con la Encuesta Nacional de Inmigración-ENI (INE, 2009c), para la provincia de Almería, respecto a la situación laboral de los encuestados. Pues, aunque hay pequeñas diferencias, se estima que se debe a la diferente coyuntura económica existente en el momento de realizar ambas encuestas.

Respecto a las ocupaciones preferidas por los inmigrantes que prestan sus servicios en la provincia de Almería, no existe concordancia entre los resultados obtenidos por la ENI y los nuestros. Consideramos que es debido a la reestructuración de los sectores productivos tras la crisis económica actual y a que el $43 \%$ de los encuestados en la ENI no declaran el sector de actividad que prestan sus servicios. Asimismo, se observa cierta similitud entre los resultados de nuestra encuestas y los de la ENI respecto a la duración del contrato de trabajo, considerando todas las modalidades de contrato indefinido obtenidos por la ENI.

Estimamos que existe concordancia entre los resultados de la ENI y los nuestros respecto al régimen de vivienda de los inmigrantes, aunque hay una variación de diez puntos porcentuales entre los que declaran residir en viviendas de su propiedad y alquiler entre los resultados de ambas encuestas. Consideramos que se debe al incremento de los inmigrantes durante el periodo transcurrido desde el año 2007 hasta 2010 .

Finalmente, los resultados de nuestras encuestas difieren de los de la ENI respecto a los ingresos medios mensuales obtenidos por los extranjeros. Consideramos que estas diferencias se deben a la crisis económica.

\section{REFERENCIAS}

Aznar, J.A., Belmonte, L.J. y Bonillo, D. (2009). Mercado de trabajo y población extranjera en Almería: el sector de la agricultura intensiva. Universidad de Almería.

Aznar, J.A., Belmonte, L.J., Bonillo, D., Molina, A. y Saquilán, H. (2009). Análisis de la inmigración en la provincia de Almería. Junta de Andalucía-Consejería de Gobernación. Delegación de Gobierno de Almería.

Aznar, J.A., Belmonte, L.J. y Bonillo, D. (2008). La inmigración en el sur de España: la provincia de Almería, ¿un mercado de trabajo de paso? Trabajo: Revista Andaluza de Relaciones Laborales, 21, 181-200.

Belmonte, L.J, Corrales, A.J. y Ruiz, J. (2009). Sostenibilidad del sistema de pensiones en España. European Journal of Education and Psychology, 2(2), 113-129.

De la Rica, S. (2010). Inmigración. En M. Bagúes, J. Fernández-Villaverde y L. Garicano. La Ley de Economía Sostenible y las reformas estructurales: 25 propuestas. FEDEA, 140-145. Madrid. Disponible en Internet: www.fedea.es. 
Dolado, J.J. y Vázquez, P. (2008). Ensayos sobre los efectos económicos de la inmigración en España. Colección de Estudios Económicos 01-08, FEDEA. Madrid. Disponible en Internet: www.fedea.es.

Instituto Nacional de Estadística-INE (2005). Estudio descriptivo de la pobreza en España. Resultados basados en la Encuesta de Condiciones de Vida 2004. Análisis Sociales: Análisis de la renta y el gasto de los hogares, Madrid. Disponible en Internet: http://www.ine.es/daco/daco42/sociales/estudiodesc.pdf

Instituto Nacional de Estadística-INE (2009). Encuesta de Condiciones de Vida de 2008, Boletín Mensual de Estadística, Octubre de 2009, Madrid.

Instituto Nacional de Estadística - INE (2009b). Encuesta de Población Activa. Diseño de la Encuesta y Evaluación de la calidad de los datos. Madrid: INE. Disponible en http://www.ine.es/docutrab/epa05_disenc/epa05_disenc.pdf

Instituto Nacional de Estadística-INE (2009c). Encuesta Nacional de Inmigrantes 2007: una monografía. Madrid.

Observatorio Permanente de la Inmigración (2010): Informe Estadístico Trimestral-Febrero 2010, Secretaría de Estado de Inmigración y Emigración, Ministerio de Trabajo e Inmigración, Madrid (edición electrónica).

Pajares, M. (2009). Inmigración y mercado de trabajo. Informe 2009. Observatorio Permanente de la Inmigración-Secretaría de Estado de Inmigración y Emigración del Ministerio de Trabajo e Inmigración. Madrid. 
